\title{
INTRODUCTIONS DE NOUVELLES ESPÈCES DE POISSONS DANS LES EAUX DOUCES TROPICALES : OBJECTIFS ET CONSÉQUENCES.
}

\author{
C. LÉVÊQUE
}

ORSTOM, 213 rue La Fayette, 75480 Paris Cedex 10, France.

\section{RÉSUMÉ}

Les introductions d'espèces dans les milieux aquatiques tropicaux ont d'abord eu pour motivation de développer la pêche sportive pour les émigrants européens. Actuellement, les principaux objectifs sont d'améliorer la pêche artisanale locale et de développer l'aquaculture. L'introduction de poissons pélagiques de la famille des clupéidés dans divers lacs naturels ou de barrage apparaît comme un succès, car elle a permis de développer la pêche de manière significative, sans dommages apparents pour la faune indigène. Par contre, l'introduction de prédateurs a toujours eu des conséquences négatives sur la faune autochtone, et divers exemples sont donnés dont la truite (Salmo trutta Linnaeus, 1758) en Australie et dans le lac Titicaca, et le capitaine (Lates niloticus Linnaeus, 1758) dans le lac Victoria. Les introductions de tilapias donnent lieu à des bilans plus mitigés, généralement positifs lorsque les cichlidés sont introduits dans des milieux pauvres en espèces autochtones, alors que des disparitions d'espèces ont été constatées pour les introductions réalisées dans les milieux déjà riches en espèces. L'exemple des lacs malgaches montre les conséquences d'introductions successives de diverses espèces sur la faune ichtyologique et la pêche.

\section{INTRODUCTIONS OF EXOTIC FISH SPECIES IN TROPICAL FRESHWATERS : PURPOSES AND CONSEQUENCES.}

\begin{abstract}
European emigrants introduced several fish species into tropical freshwater ecosystems for the purpose of sport fisheries. At present, the main goals of introductions are the management of small-scale fisheries, and aquaculture. The introduction of clupeid pelagic species into natural or artificial lakes was an economic success, because it allowed an improvement of the local fisheries while no adverse impacts on the indigenous fauna were reported. On the contrary, the introduction of large predators has had dramatic consequences on the native fauna. This was the case for trout (Salmo trutta Linnaeus, 1758) in Australia or Lake Titicaca, and the Nile-perch (Lates niloticus Linnaeus, 1758) in Lake Victoria. Tilapiines may be beneficial to the ecosystem and local fisheries when introduced into depauperated systems, while in systems with greater species richness, the disappearance of fish species following the introduction of cichlids was reported. Madagascar lakes provide good examples of the impact of successive introductions of various fish species on native fish assemblages and fisheries.
\end{abstract}

\section{INTRODUCTION}

Les introductions d'espèces de poissons en milieu tropical ont des objectifs souvent différents des préoccupations métropolitaines, tout en offrant des modèles écologiques parfois exceptionnels qu'il n'est pas possible de rencontrer en Europe. Cet article tente d'illustrer l'expérience acquise en milieu tropical, sur la base de quelques exemples sélectionnés, afin d'apporter quelques éléments supplémentaires de réflexion au problème des introductions. 


\section{OBJECTIFS DES INTRODUCTIONS D'ESPĖCES EN MILIEU TROPICAL}

\section{Développer la pêche sportive}

Plusieurs espèces de salmonidés ont été introduites, en différents endroits du monde, pour la pêche sportive. Un exemple spectaculaire est celui de la truite commune (Salmo trutta Linnaeus, 1758), originaire d'Europe, qui est maintenant présente sur tous les continents (Fig. 1) (BAGLINIĖRE et MAISSE, 1991). Les premières transplantations ont débuté au milieu du 19 ème siècle, sachant que l'introduction de la truite commune, comme celle de la carpe d'ailleurs, fut en grande partie motivée par le désir des immigrants européens de retrouver un environnement et des modes de vie similaires à ceux de leur pays d'origine. Avec l'introduction de la truite, ils pouvaient ainsi pratiquer, loin de chez eux, un de leurs sports favoris.

La majorité des introductions a parfaitement réussi dans les biotopes présentant des conditions écologiques similaires à celles de l'aire d'origine, en raison des grandes capacités d'adaptation de l'espèce à la diversité des milieux.

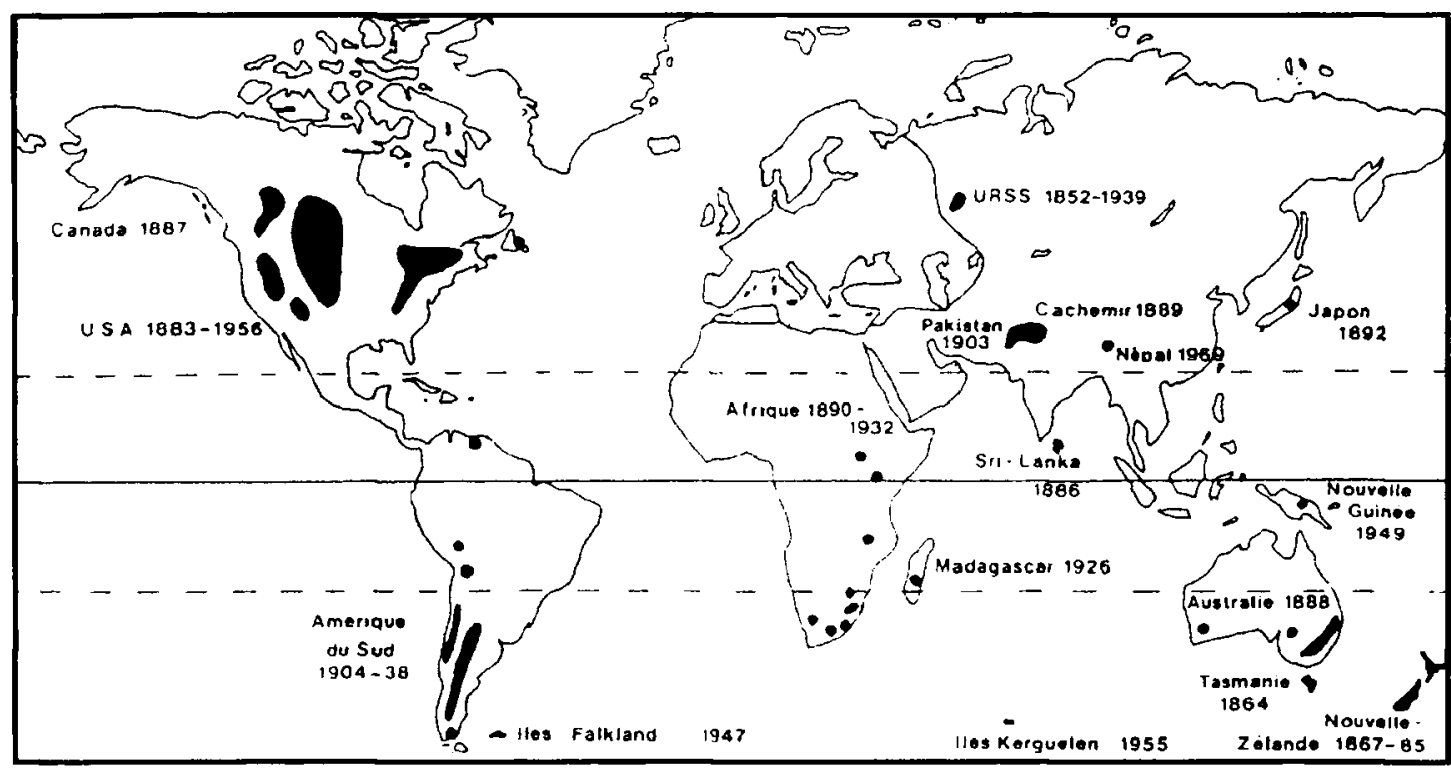

Figure 1

Introduction de la truite dans différentes régions du monde (d'après BAGLINIĖRE et MAISSE, 1991).

Figure 1

Introduction of trout (Salmo trutta) into different parts of the world (from BAGLINIERE and MAISSE, 1991).

\section{Améliorer la pêche artisanale}

Les peuplements de poissons dans les eaux tropicales sont généralement suffisamment divers pour que la plupart des niches écologiques soient utilisées. Mais, dans certains hydrosystèmes, il peut exister une faune appauvrie pour des raisons historiques. En outre, la création de grandes retenues crée de nouveaux habitats lacustres qui ne sont pas toujours colonisés par la faune fluviale. Pour justifier les introductions, les gestionnaires des pêches mettent en avant des exemples jugés comme positifs pour le développement de la pêche.

Un premier ensemble d'introductions concerne les milieux pélagiques. Limnothrissa miodon (Boulenger, 1906) et Stolothrissa tanganyicae (Regan, 1917) sont des petits Clupeidae pélagiques et zooplanctivores du lac Tanganyika, où ils font l'objet d'une pêcherie importante. L. miodon a été introduit en 1967 et 1968 dans le lac Kariba, un grand lac de barrage sur le Zambèze, et s'y est très bien développé (Fig. 2) au point d'alimenter également une pêcherie 
(MARSHALL, 1984). L'espèce a par la suite colonisé un autre barrage, celui de Cahora Bassa, en aval de Kariba. L. miodon et S. tanganyicae ont également été introduits dans le lac Kivu en 1958-1960. Ici encore, ils ont occupé la niche vacante de pélagique zooplanctivore, et on estime que cette introduction est un succès économique, sans que des impacts négatifs aient été relevés (SPLIETHOFF et al., 1983).

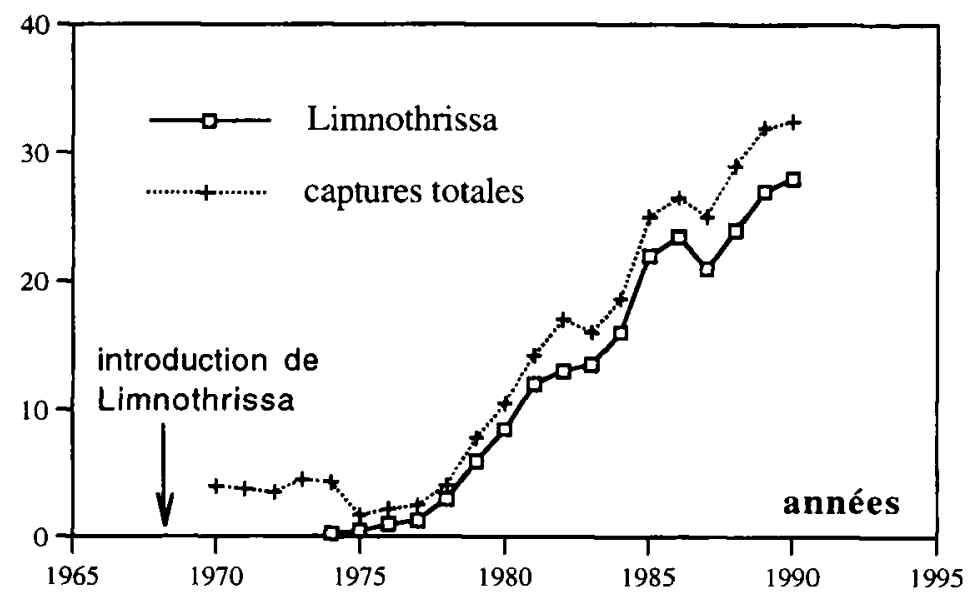

\section{Figure 2}

Évolution de la pêche dans le lac Kariba depuis l'introduction du clupeidé pélagique, Limnothrissa miodon (données d'après CRUL et ROEST, 1995).

Figure 2

Changes in the yearly fish catch in Lake Kariba since the introduction of the pelagic clupeid, Limnothrissa miodon (data from CRUL and ROEST, 1995).

L'introduction de diverses espèces de tilapias d'origine africaine (en particulier, Oreochromis mossambicus Peters, 1852) dans les lacs naturels et les réservoirs artificiels a également permis d'améliorer la pêche de subsistance dans de nombreux pays asiatiques (FERNANDO, 1991 ; FERNANDO et FURTADO, 1975 ; HOLCIK, 1984). Les tilapias (Oreochromis $s p$. et Sarotherodon $s p$.) sont de très bonnes espèces colonisatrices, en raison à la fois de leurs caractéristiques biologiques (espèces pratiquant des soins parentaux), de leur grande tolérance physiologique, et de leur capacité à se nourrir d'algues et de détritus qui sont des ressources souvent mal exploitées dans beaucoup de systèmes tropicaux (PHILIPPART et RUWET, 1982). Dans le lac Parakrama Samudra au Sri Lanka, la pêche, qui n'était que $1 \mathrm{~kg} \mathrm{ha}^{-1}$ avant 1953, atteint $445 \mathrm{~kg} \mathrm{ha}^{-1}$ en 1978, après l'introduction d'O. mossambicus. Des exemples similaires existent pour d'autres milieux d'Asie du sud-est. Au Sri Lanka, les tilapias introduits forment maintenant une composante essentielle des pêcheries dans les réservoirs (COSTA, 1994).

Dans des systèmes aquatiques possédant une faune piscicole appauvrie, les introductions ont été justifiées afin d'améliorer la production de la pêche. C'est le cas pour la rivière Sepik en Papouasie (Nouvelle-Guinée), dont la faune est composée essentiellement d'espèces estuariennes et catadromes (HAINES, 1983). Partant de l'hypothèse qu'il existait des niches non occupées et qu'il était possible d'augmenter la production piscicole, le Cichlidae Oreochromis mossambicus a été introduit au milieu des années 1950 et constitue maintenant environ $50 \%$ de la production piscicole. La carpe commune s'est également établie en certains endroits. Ces introductions ont eu lieu sans connaissance préalable de la faune autochtone, de telle sorte qu'il est difficile d'évaluer les conséquences de ces introductions. Cependant, l'amélioration significative de la pêche par l'introduction de nouvelles espèces a bénéficié aux populations locales souffrant de malnutrition (COATES, 1987). 
Oreochromis niloticus (Linnaeus, 1758) a été introduit dans de nombreux plans d'eau naturels ou artificiels en Afrique, en Asie et en Amérique du sud, et notamment dans les nombreux petits barrages qui ont èté construits au cours des deux dernières décennies. Plusieurs espèces de tilapias sont implantées actuellement dans toute la ceinture intertropicale. En Afrique, Lates niloticus (Linnaeus, 1758) et Oreochromis niloticus ont été introduits dans le lac Kyoga au cours des années 1950 et ont rapidement prospéré. En 1977, ils constituaient chacun environ $40 \%$ de la pêche artisanale estimée à 167000 tonnes, alors qu'elle n'était que de 4500 tonnes en 1956. En 1985, la situation était un peu différente avec O. niloticus constituant $78 \%$ des captures contre seulement $17 \%$ pour L. niloticus (OGUTU-OHWAYO, 1990). Ces introductions se sont accompagnées d'une régression importante des espèces autochtones (tableau I).

\section{Tableau I}

Quantités de poissons pêchées (en tonnes) et composition des captures dans le lac Kyoga, entre 1963 et 1988, montrant la contribution des espèces introduites. (Données de OGUTU-OHWAYO, 1990).

\section{Table I}

Composition and volume of fish catches (in tons) in Lake Kyoga between 1963 and 1988, showing the contribution of introduced species. (Data from OGUTU-OHWAYO, 1990).

\begin{tabular}{|c|c|c|c|c|c|}
\hline années & $\begin{array}{c}\text { captures totales } \\
\text { (tonnes) }\end{array}$ & L. niloticus & O. niloticus & $\begin{array}{c}\text { tilapias } \\
\text { autochtones }\end{array}$ & autres \\
\hline & & & & & \\
1963 & 20000 & 2 & 3 & 37 & 58 \\
1965 & 21000 & 24 & 3 & 20 & 53 \\
1967 & 26000 & 48 & 19 & 11 & 22 \\
1970 & 63000 & 52 & 26 & 4 & 18 \\
1973 & 97000 & 57 & 32 & 0 & 11 \\
1975 & 121000 & 43 & 42 & 0 & 15 \\
1978 & 162000 & 42 & 41 & 0 & 17 \\
1985 & 103000 & 17 & 78 & & 5 \\
1986 & 57000 & 4 & 91 & & 5 \\
1988 & & 13 & 86 & & 1 \\
& & & & & \\
\hline
\end{tabular}

\section{Développer l'aquaculture}

Plus d'une centaine d'espèces de poissons ont fait l'objet d'introductions à des fins d'aquaculture, mais moins d'une dizaine ont été largement diffusées dans le monde. Parmi celles-ci, la carpe commune (Cyprinus carpio Linnaeus, 1758), originaire d'Asie, est probablement la première espèce qui ait fait l'objet de transferts importants, puisque les romains prélevèrent des exemplaires sauvages dans le Danube et répandirent par la suite cette espèce à travers l'Europe, où son élevage fit partie, dès le Moyen-Age, de la tradition monastique (BALON, 1995). Depuis le milieu du siècle dernier, la carpe a été introduite dans la plupart des régions du monde où les conditions climatiques permettent sa survie. La carpe herbivore, Ctenopharyngodon idella Cuvier et Valenciennes, 1821, a connu un succès similaire.

Un autre groupe de poissons qui a connu également un grand succès en aquaculture, au point d'être maintenant présent dans toute la zone intertropicale. est celui des tilapias (Cichlidae). Oreochromis niloticus et, dans une moindre mesure, Oreochromis mossambicus, originaires d'Afrique, constituent à l'heure actuelle la principale production aquacole du sud-est asiatique. Ces espèces, ou d'autres espèces de la famille des tilapias, sont maintenant présentes en Amérique du sud et dans le sud des États-Unis, et même en Europe où elles sont élevées dans des eaux réchauffées (Fig. 3). 


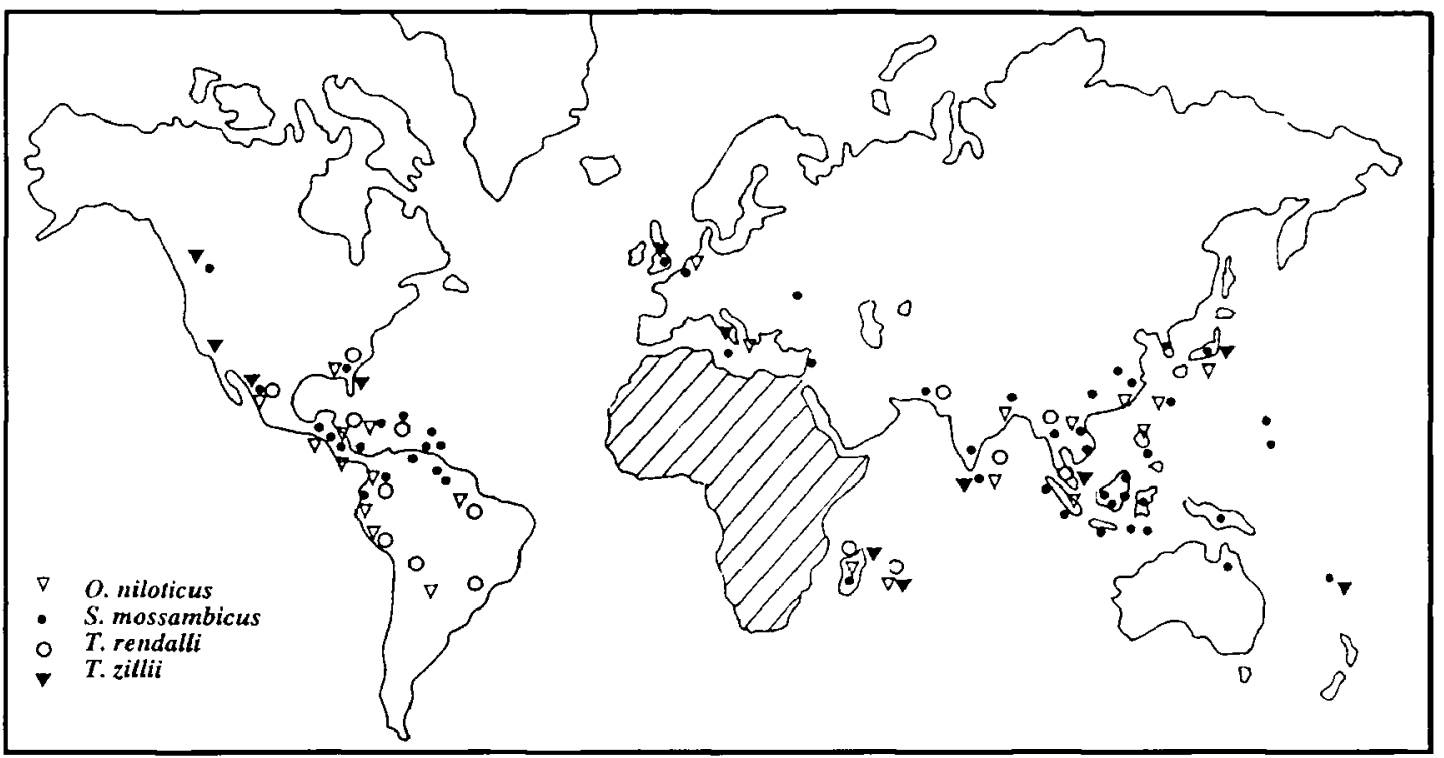

\section{Figure 3}

Distribution actuelle de quelques espèces de cichlidés d'origine africaine, introduites dans diverses régions du monde.

Figure 3

Present-day distribution of several cichlid species native from Africa and introduced into various parts of the world.

\section{Contrôle biologique}

L'introduction d'espèces de poissons a été pratiquée pour lutter contre les vecteurs de maladies. Différentes espèces de poissons, comme Gambusia affinis Baird et Girard, 1853 ou Poecilia reticulata Peters, 1859 , ont été utilisées pour contrôler les moustiques, vecteurs du paludisme. On a également proposé d'utiliser le cichlidé malacophage Astatoreochromis alluaudi Pellegrin, 1904, originaire d'Afrique de l'est, pour lutter contre les vecteurs de bilharziose (SLOOTWEG, 1989). Cette espèce a été introduite en différents endroits d'Afrique sans que des effets écologiques n'aient été enregistrés, mais sans que son efficacité, en tant qu'agent de contrôle, n'ait été réellement prouvée non plus. Enfin, des poissons africains sont parfois utilisés pour lutter contre la végétation aquatique, comme Tilapia rendalli (Boulenger, 1896) qui a été introduit dans des canaux d'irrigation au Soudan.

\section{CONSÉQUENCES DES INTRODUCTIONS D'ESPÈCES}

Les espèces introduites peuvent prendre place dans l'écosystème, sans perturbation majeure apparente de ce dernier. Mais lorsqu'on dispose d'observations détaillées, ce qui n'est pas toujours le cas, on observe fréquemment des modifications dans les systèmes biologiques qui peuvent aller jusqu'à la disparition de plusieurs espèces autochtones.

\section{Prédation des espèces introduites sur les espèces autochtones}

Les introductions de truites ont eu des répercussions sur les espèces de poissons indigènes. En Australie, par exemple, l'introduction de Salmo trutta a provoqué l'éradication de certaines espèces de Galaxidae en raison d'une forte prédation (JACKSON et WILLIAMS, 1980).

En Amérique du sud, quatre espèces de Salmonidae, Salvelinus namaycush (Walbaum, 1794), Salvelinus fontinalis (Mitchill, 1815), Salmo trutta et Oncorhynchus mykiss (Walbaum, 1792) ont èté introduites avec des succès divers dans le lac Titicaca, où seulement 
Oncorhynchus mykiss s'est acclimaté (LOUBENS, 1989). Ce Salmonidae paraît être responsable de la disparition de quelques espèces d'Orestias, comme Orestias cuvieri Valenciennes, 1846, ainsi que de la raréfaction d'autres espèces.

En Afrique, on estime également que c'est l'introduction du black-bass, Micropterus salmoides Lacépède, 1802, dans le lac Naivasha en 1929 pour la pêche sportive, qui serait responsable de la disparition des quelques espèces indigènes, ainsi que des espèces introduites pour lutter contre les larves de moustiques, comme Gambusia et Poecilia sp. L'un des cas les plus spectaculaires est celui de l'introduction dans le lac Victoria du Lates niloticus, un poisson piscivore pouvant atteindre plus de $100 \mathrm{~kg}$ (voir ci-dessous).

\section{Compétition avec les espèces indigènes}

Les espèces introduites peuvent entrer en compétition avec les espèces indigènes, et éventuellement les éliminer, lorsqu'elles utilisent les mêmes ressources trophiques. Ainsi, dans le lac Luhondo (Ruanda), trois espèces de cyprinidés étaient connues jusqu'en 1934: un petit barbeau, Barbus neumayeri Fischer, 1884, et deux grandes espèces, Barbus microbarbis David et Poll, 1937 et Varicorhinus ruandae Pappenheim et Boulenger, 1914 (DE VOS et al., 1990). Oreochromis niloticus a été introduit volontairement en 1935, ainsi que quelques Haplochromis de manière apparemment accidentelle. $O$. niloticus était devenu l'espèce dominante dans les années 1950, puis les Haplochromis se sont à leur tour développés au point d'être maintenant les plus abondants. Par contre, B. microbrabis a disparu et les deux autres cyprinidés sont maintenant cantonnés aux petits cours d'eau tributaires du lac.

\section{Cascades trophiques et conséquences sur les écosystèmes aquatiques}

L'introduction d'un prédateur dans un écosystème aquatique peut avoir des conséquences sur le fonctionnement biologique du système, par le biais des chaines trophiques (LÉVÊQUE, 1995). ZARET et PAINE (1973) ont étudié les conséquences de l'introduction du poisson piscivore Cichla ocellaris Bloch et Scneider (cichlidé) sur le fonctionnement écologique du lac Gatun (Panama). II y a eu une diminution importante de l'abondance des consommateurs secondaires, ainsi que des prédateurs de petits poissons comme les hérons et les martins-pêcheurs.

Un autre exemple spectaculaire de cascade trophique est celui consécutif à l'introduction du capitaine (Lates niloticus) dans le lac Victoria. Ce poisson piscivore, qui peut atteindre $100 \mathrm{~kg}$, a provoqué en quelques années la disparition de plusieurs espèces de cichlidés endémiques. Ainsi, on a constaté la quasi-disparition dans les années 1980 du groupe détritivore/phytoplanctivore des haplochromines (cichlidés endémiques), ainsi que du groupe des zooplanctivores, qui constituaient respectivement 40 et $16 \%$ de la biomasse de poissons démersaux. Ils ont été remplacés par la crevette indigène Caridina nilotica Roux, 1833 et par le cyprinidé Rastineobola argentea (Pellegrin, 1904) (WITTE et al., 1992a et b), ces deux dernières espèces étant devenues la nourriture principale des capitaines après la disparition des haplochromines. L'introduction du capitaine a donc entraîné une simplification des chaînes trophiques, et ce prédateur mange ses propres juvéniles qui, d'une certaine manière, remplissent la même fonction de zooplanctivore que les haplochromines auparavant. Une autre conséquence a été une augmentation importante des insectes aquatiques, dont les adultes forment à certaines époques de véritables nuages au-dessus du lac. Cette population d'insectes sert à son tour de nourriture à l'hirondelle Riparia riparia (Linnaeus), qui hiverne en Afrique et dont la population s'est accrue massivement (SUTHERLAND, 1992). Une autre conséquence de la diminution des haplochromines et de l'augmentation des Rastineobola a été le changement du régime alimentaire du martin-pêcheur Ceryle rudis (Linnaeus) qui, actuellement, consomme essentiellement ce cyprinidé pélagique (WANINK et GOUDSWAARD, 1994). II semble qu'un changement similaire dans l'alimentation soit intervenu chez le cormoran Phalacrocorax carbo. 


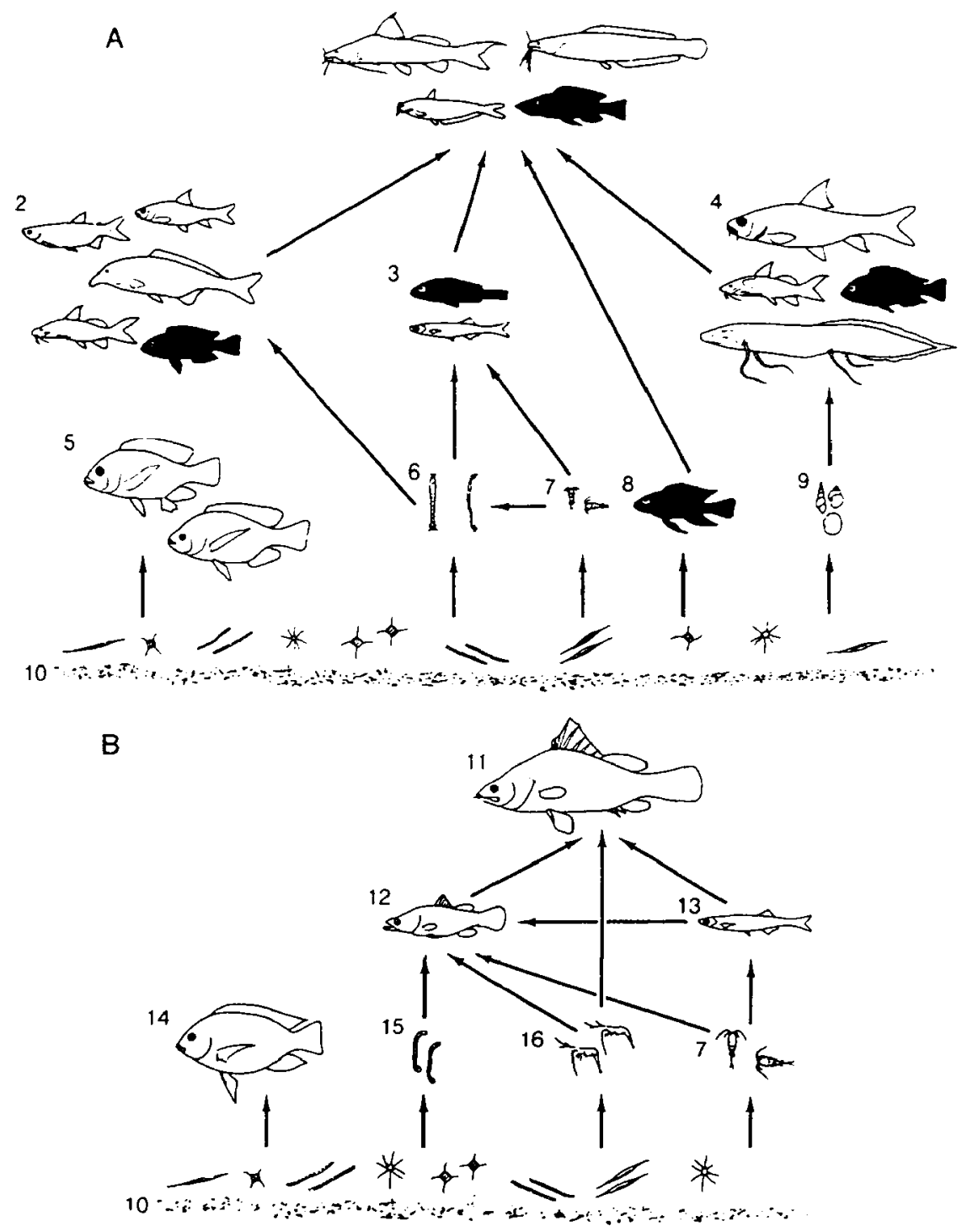

Figure 4

Représentation simplifiée des chaînes trophiques dans les populations de poissons démersaux du lac Victoria, avant et après introduction des Lates (d'après WITTE et al., 1992b). Seuls les organismes qui constituent une part importante de l'alimentation des principales espèces de poissons on été mentionnés. En 1970, les Haplochromis (qui sont figurés en noir) étaient dominants en nombre et en biomasse dans tous les groupes trophiques, à l'exception des piscivores.

Figure 4

Simplified food-webs of demersal fish populations in Lake Victoria, before and after introduction of Lates (from WITTE et al., 1992b). Only food items contributing significantly to the diet are mentioned. In 1970, the Haplochromis (in black on the figure) were dominant in number and biomass in every group, except for piscivores.

1 : Bagrus docmac, Clarias gariepinus, Schilbe mystus, Haplochromis piscivores. 2 : Brycinus spp., Barbus spp., Mormyridae, Synodontis afrofischeri, Haplochromis insectivores. 3 : Haplochromis zooplanctivores, Rastrineobola argentea. 4 : Barbus altianalis, Synodontis victoriae, Haplochromis molluscivores. 5 : Oreochromis variabilis, 0. esculentus. 6 : Chaoborus spp., chironomides. 7 : zooplancton. 8 : Haplochromis détritivores ou phytoplanctivores. 9 : mollusques. 10 : détritus, phytoplancton. 11 : Lates niloticus. 12 : juvéniles de Lates. 13 : Rastrineobola argentea. 14 : Oreochromis niloticus. 15 : chironomides. 16 : Caridina nilotica (d'après LIGVOET et WITTE, 1991 et WITTE at al., 1992b). 

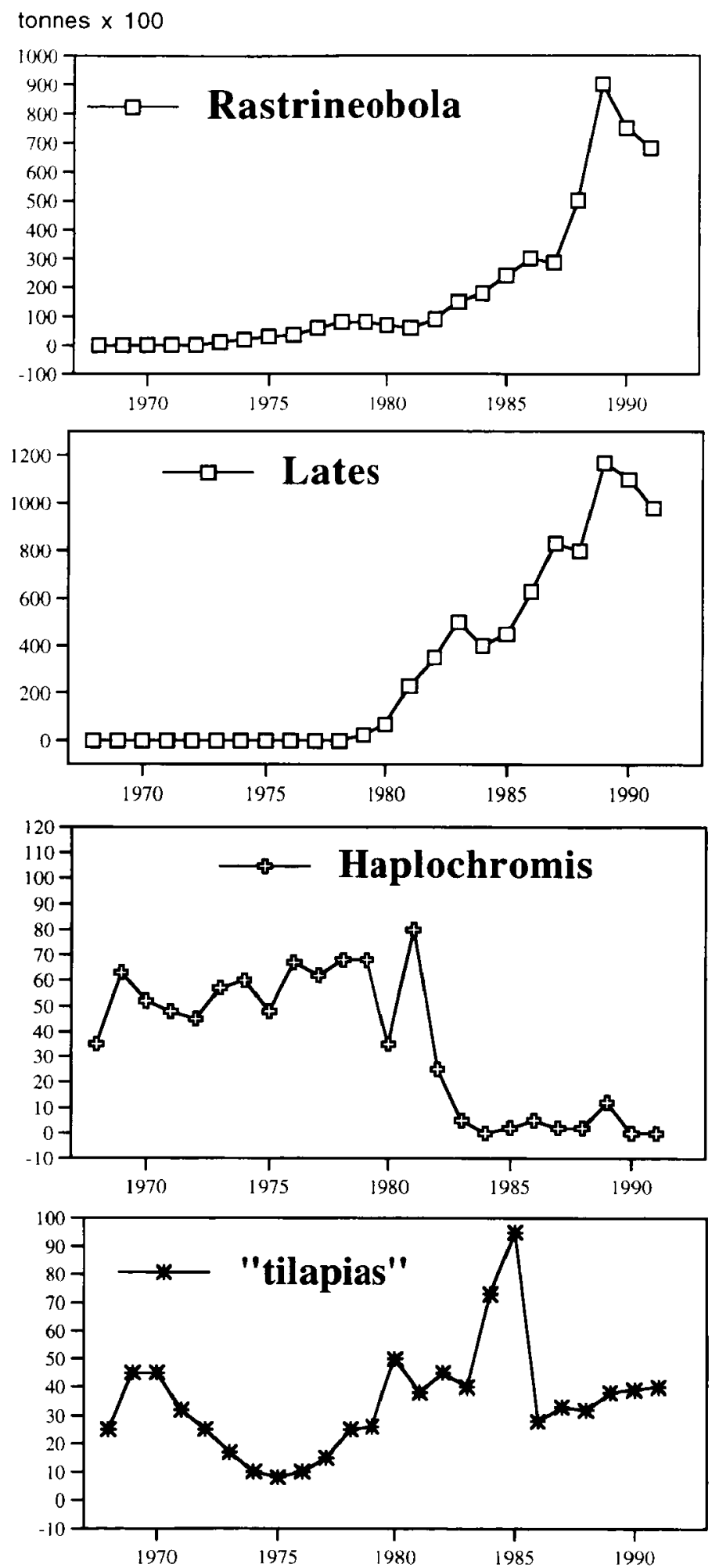

Figure 5

Changements intervenus dans l'abondance de différentes espèces de poissons dans les captures commerciales, dans la partie kenyanne du lac Victoria, après le développement du Lates, introduit dans les années 1960 (d'après GOPHEN et al., 1995).

Figure 5

Changes in the abundance of various fish species in commercial catches, in the Kenyan side of Lake Victoria, when Lates introduced in the 1960s expanded (from GOPHEN et al., 1995). 
On peut comparer les chaines trophiques ci-dessus à celles suggérées il y a plus de 60 ans par WORTHINGTON (1933). Une différence essentielle réside dans la disparition des crocodiles qui étaient très abondants dans le lac Victoria, et qui a certainement entraîné elle aussi de profonds changements dans le fonctionnement de l'écosystème, lesquels n'ont pu malheureusement être observés. Une question que l'on peut se poser est de savoir si l'introduction du Lates aurait eu les mêmes conséquences, si les crocodiles n'avaient pas disparu?

Un autre exemple de cascade trophique est celui de l'introduction d'Oreochromis alcalinus grahami (Boulenger, 1912) dans le lac Nakuru (Kenya). Dans ce lac salé, le cichlidé introduit dans les années cinquante s'est développé rapidement en consommant l'abondante biomasse de la cyanobactérie Spirulina platensis. Le développement de la population de poissons a permis l'installation d'abondantes colonies d'oiseaux piscivores (VARESCHI et JACOBS, 1984).

\section{malgaches}

Conséquences d'introductions répétées sur la faune et la pêche : le cas des lacs

La faune piscicole malgache est assez pauvre, mais constituée de nombreuses espèces endémiques appartenant en particulier à la famille des Cichlidae et à celle des Bedotiidae (REINTHAL et STIASSNY, 1991). L'introduction d'espèces étrangères a commencé au siècle dernier (KIENER, 1963; MOREAU, 1979) et les peuplements actuels de nombreux lacs malgaches (lac Alaotra, lac Itasy, par exemple) sont constitués d'espèces provenant de divers continents (tableau II). Ces espèces cohabitent parfaitement et sont à l'origine d'une pêcherie assez prospère. Mais elles ont également éliminé une partie de la faune indigène.

\section{Tableau II}

Liste des espèces introduites dans les eaux douces de Madagascar, origine géographique et date connue d'introduction.

Table II

List of introduced species into Malagasy freshwaters, origin of fish and date of introduction.

\begin{tabular}{|c|c|c|c|c|}
\hline années & espèces & provenant de & adaptées & références \\
\hline $\begin{array}{l}1857 \\
1861 \\
1914 \\
1922 \\
1926 \\
1929 \\
1940 \\
1951 \\
1951 \\
1951 \\
1955 \\
1955 \\
1956 \\
1956 \\
1959 \\
1963 \\
1975\end{array}$ & $\begin{array}{l}\text { Osphronemus goramy } \\
\text { Carassius auratus } \\
\text { Cyprinus carpio } \\
\text { Salmo irideus } \\
\text { Salmo fario } \\
\text { Gambusia holbrooki } \\
\text { Oncorhynchus mykiss } \\
\text { Tilapia melanopleura } \\
\text { Micropterus salmoides } \\
\text { Tilapia rendalli } \\
\text { Tilapia zillii } \\
\text { Oreochromis macrochir } \\
\text { Oreochromis mossambicus } \\
\text { Oreochromis niloticus } \\
\text { Cyprinus carpio } \\
\text { Heterotis niloticus } \\
\text { Ophicephalus striatus }\end{array}$ & $\begin{array}{c}\text { Asie } \\
\text { France } \\
\text { France } \\
\text { USA } \\
\text { France } \\
\text { USA } \\
\text { Réunion } \\
\text { Congo } \\
\text { Amérique du nord } \\
\text { Congo } \\
\text { Kenya } \\
\text { Congo } \\
\text { Mozambique } \\
\text { Egypte } \\
\text { France } \\
\text { RCA } \\
\text { Asie }\end{array}$ & $\begin{array}{l}\text { Pangalanes } \\
\text { répandue } \\
\text { répandue } \\
\text { Ankaratra } \\
\text { Ankaratra } \\
\text { répandue } \\
\text { répandue } \\
\text { altitudes moyennes } \\
\text { en quelques endroits } \\
\text { répandue } \\
\text { répandue } \\
\text { répandue } \\
\text { Pangalanes } \\
\text { différents lacs }\end{array}$ & $\begin{array}{c}\text { Kiener, } 1963 \\
\text { Kiener, } 1963 \\
\text { Kiener, } 1963 \\
\text { Kiener, } 1963 \\
\text { Kiener, } 1963 \\
\text { Kiener, } 1963 \\
\text { Moreau, } 1988 \\
\text { Kiener, } 1963 \\
\text { Kiener, } 1963 \\
\text { Moreau, } 1982 \\
\text { Kiener, } 1963 \\
\text { Kiener, } 1963 \\
\text { Kiener, } 1963 \\
\text { Kiener, 1963 } \\
\text { Kiener, 1963 } \\
\text { Moreau, } 1988 \\
\text { Rabelahatra, } 1988\end{array}$ \\
\hline
\end{tabular}

Dans le lac Alaotra, avant l'introduction d'espèces exotiques, la communauté piscicole était pauvre et dominée par Paratilapia polleni (Bleeker, 1868), Rheocles alaotrensis (Pellegrin, 1914), Ratsirakia legendrei (Pellegrin, 1919), et deux anguilles (Anguilla mossambica (Peters, 1852) et A. marmorata (Quoy et Gaimard, 1824)) (MOREAU, 1980). Au début du siècle, le 
Carassius auratus (Linnaeus, 1758), introduit à Madagascar en 1865, est signalé du lac et, en 1925, P. polleni ainsi que Carassius auratus constituaient l'essentiel des captures de la pêche artisanale (tableau III). En 1935, le Cyprinus carpio introduit en 1925 est une composante non négligeable des captures et, en 1954, cette espèce est largement dominante (tableau III).

\section{Tableau III}

Évolution de la composition des captures de la pêche artisanale dans le lac Alaotra (Madagascar), en fonction des introductions successives d'espèces (d'après MOREAU, 1980).

Table III

Changes in the catch composition of artisanal fisheries in Lake Alaotra (Madagascar), as a result of successive species introductions (from MOREAU, 1980).

\begin{tabular}{|c|c|c|c|c|c|c|c|c|c|c|}
\hline années & 1925 & 1935 & 1954 & 1957 & 1960 & 1963 & 1966 & 1969 & 1972 & 1975 \\
\hline $\begin{array}{l}\text { captures totales } \\
\% \text { dans les captures }\end{array}$ & & & 1800 & 2200 & 2700 & 3000 & 3200 & 2500 & 2100 & 2100 \\
\hline $\begin{array}{l}\text { Anguilla spp. } \\
\text { Paratilapia polleni }\end{array}$ & $\begin{array}{c}5 \\
75\end{array}$ & $\begin{array}{c}5 \\
40\end{array}$ & $\begin{array}{l}1 \\
5\end{array}$ & $\begin{array}{l}1 \\
3\end{array}$ & $\begin{array}{l}1 \\
2\end{array}$ & $\begin{array}{l}1 \\
1\end{array}$ & 1 & 1 & 1 & 1 \\
\hline Carassius auratus & 20 & 25 & 14 & 10 & 4 & 2 & & & & \\
\hline $\begin{array}{l}\text { Cyprinus carpio (1925) } \\
\text { Tilapia rendalli (1954) }\end{array}$ & & 30 & 80 & $\begin{array}{l}40 \\
46\end{array}$ & $\begin{array}{l}15 \\
23\end{array}$ & $\begin{array}{c}14 \\
8\end{array}$ & $\begin{array}{c}5 \\
10\end{array}$ & $\begin{array}{l}11 \\
20\end{array}$ & $\begin{array}{l}21 \\
16\end{array}$ & $\begin{array}{l}21 \\
16\end{array}$ \\
\hline Oreochromis macrochir (1958) & & & & & 55 & 74 & 85 & 66 & 56 & 50 \\
\hline Oreochromis niloticus (1961) & & & & & & & & 1 & 3 & 7 \\
\hline Micropterus salmoides (1961) & & & & & & & 1 & 1 & 3 & 4 \\
\hline
\end{tabular}

Puis, vient une période durant laquelle trois espèces de tilapias sont introduites successivement, et deux d'entre elles (Tilapia rendalli et Oreochromis macrochir) prolifèrent au cours des années 1960 , alors que les espèces indigènes et $C$. auratus régressent fortement. La diminution des tilapias dans les captures des années 1970 pourrait être due à une surexploitation résultant de l'introduction des filets maillants dans la pêcherie, à la fin des années 1960. Le black-bass Micropterus salmoides, introduit en 1962, ne s'est pas beaucoup développé puisqu'il ne constituait que $4 \%$ des captures en 1975. De 1966 à 1972, des juvéniles de Cyprinus carpio ont été réintroduits dans le lac, ce qui peut expliquer un léger accroissement des captures.

Plus récemment, Ophicephalus striatus Bloch (le fibata), échappé de pisciculture, s'est répandu dans les eaux naturelles malgaches. Ce prédateur d'origine asiatique pourrait avoir un impact important sur la faune piscicole.

Une histoire similaire s'est passée dans le lac Itasy (MOREAU, 1979), qui était habité par plusieurs espèces indigènes au début du siècle : Anguilla mossambica, Anguilla bengalensis labiata (Peters, 1852), Anguilla marmorata, Ptychochromoides betsileanus (Boulenger, 1899), Ratsirakia legendrei et Chonophorus macrorhynchus (Bleeker, 1867). Carassius auratus fut introduit en 1899, et Paratilapia polleni (une espèce endémique malgache) en 1924, mais ils ne se sont pas réellement développés. En 1930, Ptychochromoides betsileanus constituait $40 \%$ des captures, mais en 1950-1955, Cyprinus carpio, qui avait été introduit en 1925-1930, constituait 80 à $85 \%$ des captures, contre $10 \%$ seulement pour P. betsileanus (tableau IV). Tilapia rendalli, introduit en 1955, remplaça rapidement Cyprinus carpio et vers 1958 représentait $70 \%$ des captures. Mais $T$. rendalli lui-même fut remplacé par Oreochromis macrochir, qui avait été introduit en 1958 et qui constitua l'essentiel de la pêche en 1963. En 1965, l'importance de O. macrochir diminue, en raison de l'apparition dans les captures d'une population hybride appelée Tilapia 3/4, et en 1972 O. macrochir avait disparu. Tilapia 3/4 est le résultat d'une hybridation naturelle entre $O$. macrochir et $O$. niloticus, qui avait été introduit en 
1961. Cette hybridation spontanée donna naissance à des hybrides fertiles plus féconds que $O$. macrochir, à taille identique. Mais les populations d'O. niloticus se développèrent lentement et en 1975 représentaient $55 \%$ des captures, suivis par Tilapia 3/4 qui commençait à présenter des signes de dégénérescence. La contribution des espèces dans les captures était sensiblement similaire en 1985 à celle de 1975 (MATTHES, 1985).

\section{Tableau IV}

Évolution de la composition des captures de la pêche artisanale dans le lac Itasy (Madagascar), en fonction des introductions successives d'espèces (d'après MOREAU, 1979).

\section{Table IV}

Changes in the catch composition of artisanal fisheries in Lake Itasy (Madagascar), as a result of successive species introductions (from MOREAU, 1979).

\begin{tabular}{|c|c|c|c|c|c|c|c|c|c|c|c|c|c|c|c|c|c|}
\hline annees & 1930 & \begin{tabular}{c|}
$1950-$ \\
1955
\end{tabular} & 1958 & 1963 & 1964 & 1965 & 1966 & 1967 & 1968 & 1969 & 1970 & 1971 & 1972 & 1973 & 1974 & 1975 & 1985 \\
\hline captures totales & & & & 1200 & 850 & 5.00 & 300 & 280 & 84 & 830 & 450 & 300 & 290 & 280 & 280 & 275 & 625 \\
\hline$\%$ dans les captures & & & & & & & & & & & & & & & & & \\
\hline Ptichochromis betsileanus & 40 & 10 & 0 & 0 & & & & & & & & & & & & & \\
\hline Anguilla spp & $50 ?$ & & & 3 & 2 & 2 & 2 & 1 & 1 & 1 & 1 & 1 & $\mathbf{1}$ & 1 & 1 & 1 & \\
\hline Cyprinus carpio (1925) & & $80-85$ & & 6 & 6 & 5 & 4 & 4 & 4 & 3 & 3 & 3 & 3 & 3 & 3 & 3 & 1.5 \\
\hline Tilapia rendalli (1955) & & & 70 & 5 & 4 & 4 & 3 & 3 & 2 & 1 & 1 & 1 & 1 & 1 & 1 & & 0.4 \\
\hline Oreochromis macrochir(1958) & & & & 85 & 86 & 78 & 56 & 30 & 10 & 1 & 1 & 0 & & & & & \\
\hline Oreochromis niloticus(1961) & & & & 1 & 2 & 5 & 8 & 10 & 15 & 18 & 24 & 32 & 39 & 45 & 50 & 55 & 57 \\
\hline Tilapia $\mathbf{3 / 4}$ & & & & 0 & 0 & 5 & 25 & 50 & 66 & 74 & 68 & 61 & 54 & 48 & 43 & 39 & 38 \\
\hline Micropterus salmoides (1961) & & & & 0 & 0 & 1 & 1 & 2 & 2 & 2 & 2 & 3 & 3 & 3 & 3 & 3 & 1.5 \\
\hline
\end{tabular}

Certaines espèces ont également été introduites, sans succès apparent, dans le lac Itasy. C'est le cas pour Micropterus salmoides introduit en 1962, Gambusia holbrooki (Girard, 1859 ) introduit vers 1950 et Oreochromis mossambicus introduit en 1961, mais ces espèces, ainsi que O. macrochir, semblent avoir disparu (MATTHES, 1985). Ophicephalus striatus a été observé dans les captures en 1985, et l'on peut penser que cette espèce prédatrice aura un impact majeur sur les populations existantes.

II faut noter que la surpêche ou, tout au moins, les changements dans les techniques de pêche ont probablement joué également un rôle dans l'évolution des populations piscicoles des lacs Alaotra et Itasy. II est cependant difficile de faire la part des conséquences liées aux introductions de celles résultant d'une évolution dans les modes d'exploitation des ressources.

\section{CONCLUSIONS}

Dans les lacs naturels dont l'origine est assez récente, ainsi que dans les réservoirs peuplés essentiellement par des poissons d'origine fluviale, il peut y avoir des niches écologiques non utilisées. En particulier, les poissons fluviaux ont généralement un comportement benthique et sont mal adaptés à coloniser les milieux pélagiques.

Parmi le grand nombre d'espèces tropicales, seuls des représentants de la famille des Cichlidae et des Clupeidae, et dans une moindre mesure des Cyprinidae, ont montré des capacités à coloniser les habitats lacustres et à améliorer de manière substantielle la production piscicole. II paraît raisonnable de limiter leur introduction aux milieux d'origine récente et d'éviter de les introduire dans des lacs anciens (FERNANDO et HOLCIK, 1982).

L'expérience des milieux tropicaux montre que l'introduction de prédateurs peut avoir des impacts assez importants sur les espèces autochtones, en particulier lorsque ces dernières sont le résultat d'une longue évolution qui a conduit à constituer des "foules d'espèces". Les introductions sont ainsi à l'origine de la perte de plusieurs de ces foules d'espèces, dans différents lacs situés sur les divers continents. 


\section{BIBLIOGRAPHIE}

BAGLINIĖRE J.L., MAISSE G., 1991. La truite : biologie et écologie. INRA Éditions, Paris, 303 p.

BALON E.K., 1995. Origin and domestication of the wild carp (Cyprinus carpio) : from Roman gourmets to the swimming flowers. In Proceedings of the second aquaculture symposium (Hungary 6-9/09/93), BILLARD R. and GALL G.A.E. (eds.), 3-48.

COATES D., 1987. Consideration on fish introductions into the Sepik River, Papua, New Guinea. Aquac. Fish. Manage., 18, 231-241.

COSTA H.H., 1994. The status of limnology in Sri Lanka. Mitt. Internat. Verein. Limnol., 24, 7385.

CRUL R.M.C., ROEST F.C., 1995. Current status of fisheries and fish stocks of the four largest African reservoirs : Kainji, Kariba, Nasser/Nubia and Volta. CIFA Technical paper, 30 , FAO, Rome.

DE VOS L., SNOEKS J., THYS VAN DEN AUDENAERDE D., 1990. The effect of Tilapia introductions in Lake Luhondo, Rwanda. Env. Biol. Fish., 27, 303-308.

FERNANDO C.H., 1991. Impacts of fish introductions in tropical Asia and America. Can. J. Fish. Aquat. Sci., 48 (suppl. 1), 24-32.

FERNANDO C.H., FURTADO J.I., 1975. Reservoir fishery resources of South-East Asia. Bull. Fish. Res. Sta. Sri Lanka, 26, 83-95.

FERNANDO C.H., HOLCIK J., 1982. The nature of fish community : a factor influencing the fishery potential and yields of tropical lakes and reservoirs. Hydrobiologia, 97, 127-140.

GOPHEN M., OCHUMBA P.B.O., KAUFMAN L.S., 1995. Some aspects of perturbations in the structure and biodiversity of the ecosystem of Lake Victoria (East Africa). Aquat. Living Resour., 8, 27-41.

HAINES A.K., 1983. Fish fauna and ecology. In PETR (ed.), The Purari - tropical environment of a high rainfall river basin. W. Junk, The Hague, Monographiae Biologicae, 51, 367-384.

HOLCIK J., 1984. Some considerations on the role of introduced species of fish in the management of inland fisheries. EIFAC Tech. Pap., 42 (suppl. vol. 2), 488-495.

JACKSON P.D., WILLIAMS W.D., 1980. Effects of brown trout, Salmo trutta L., on the distribution of some native fishes in three areas of southern Victoria. Aust. J. Freshwat. Res., 31, 61-67.

KIENER A., 1963. Poissons, pêche et pisciculture à Madagascar. Publication du Centre Technique Forestier tropical, 24, $244 \mathrm{p}$.

LÉVÊQUE C., 1995. Role and consequences of fish diversity in the functioning of African freshwater ecosystems : a review. Aquat. Living Resour., 8, 59-78.

LIGVOET W., WITTE F., 1991. Perturbation through predator introduction : effects on the food web and fish yields in Lake Victoria (East Africa). In RAVERA O. (ed.), Terrestrial and aquatic ecosystems. Perturbation and Recovery, 263-268, Ellis Horwood, New York.

LOUBENS G., 1989. Observations sur les poissons de la partie bolivienne du lac Titicaca. IV. Orestias spp., Salmo gairdneri et problèmes d'aménagements. Rev. Hydrobiol. trop., 22, 157-177.

MARSHALL B.E., 1984. Small pelagic fishes and fisheries in African inland waters. CIFA Technical paper, 14, FAO, Rome.

MATTHES H., 1985. L'état des stocks et la situation des pêches au lac Itasy. Rapport préparé pour le projet Développement des pêches continentales et de l'aquaculture. FAO, DP/MAG/82/014. 
MOREAU J., 1979. Biologie et évolution des peuplements de Cichlidae (Pisces) introduits dans les lacs malgaches d'altitude. Thèse de Doctorat d'Etat, Institut National Polytechnique, Toulouse, $345 \mathrm{p}$.

MOREAU J., 1980. Le lac Alaotra à Madagascar, 50 ans d'aménagement des pêches. Cah. ORSTOM, sér. Hydrobiol., 13, 171-179.

OGUTU-OHWAYO R., 1990. The decline of the native fishes of lakes Victoria and Kyoga (East Africa) and the impact of introduced species, especially the Nile perch, Lates niloticus, and the Nile tilapia, Oreochromis niloticus. Env. Biol. Fish., 27, 81-96.

PHILIPPART J.C., RUWET J.C., 1982. Ecology and distribution of Tilapias. In PULLIN R.V.S. and LOWE-MCCONNELL R. (eds.), The biology and culture of tilapias. ICLARM Conference Proceedings, 7, 15-59, ICLARM, Manila, Philippines.

REINTHAL P.N., STIASSNY M.L.J., 1991. The freshwater fishes of Madagascar : a study of an endangered fauna with recommendations for a conservation strategy. Conserv. Biol., 5 (2), 231-243.

SLOOTWEG R., 1989. Proposed introduction of Astatoreochromis alluaudi, an East African mollusc-crushing cichlid, as a means of snail control. Ann. Mus. r. Afri. centr., Sci. Zool., 257, 61-64.

SPLIETHOFF P.C., DE IONGH H.H., FRANK V.C., 1983. Success of the introduction of the freshwater clupeid, Limnothrissa miodon (Boulenger), in lake Kivu. Fish. Manage., 14, 17-31.

SUTHERLAND W.J., 1992. Genes map the migratory route. Nature, 360, 625-626.

VARESCHI E., JACOBS J., 1984. The ecology of Lake Nakuru (Kenya). V. Production and consumption of consumer organisms. Oecologia, 61, 83-98.

WANINK J.H., GOUDSWAARD P.C., 1994. Effects of Nile perch (Lates niloticus) introduction in Lake Victoria, East Africa, on the diet of the pied kingfisher (Ceryle rudis). Hydrobiologia, 279-280, 367-373.

WITTE F., GOLDSCHMIDT T., GOUDSWAARD P.C., LIGTVOET W., VAN OIJEN M.P.J., WANINK J.H., 1992a. Species extinction and concomitant ecological changes in Lake Victoria. Neth. J. Zool., 42, 214-232.

WITTE F., GOLDSCHMIDT T., WANINK J., VAN OIJEN M., GOUDSWAARD K., WITTE-MAAS E., BOUTON N., 1992b. The destruction of an endemic species flock : quantitative data on the decline of the haplochromine cichlids of Lake Victoria. Env. Biol. Fish., 34, 1-28.

WORTHINGTON E.B., 1933. Inland waters of Africa. The results of two expeditions to the great lakes of Kenya and Uganda, with accounts of their biology, native tribes and development. Mac Millan \& Co, London, 259 p.

ZARET T.M., PAINE R.T., 1973. Species introductions in a tropical lake. Science, 182, 449-455. 